\title{
On implementing timing-accurate computer-based experiments
}

A commentary on "The timing mega-study: comparing a range of experiment generators, both lab-based and online"

(Bridges et al., 2020)

Florian Krause $e^{1} \&$ Oliver Lindemann ${ }^{2}$

${ }^{I}$ Donders Institute for Brain, Cognition and Behaviour, Radboud University Medical Center, Nijmegen, The Netherlands

${ }^{2}$ Department of Psychology, Education and Child Studies, Erasmus University Rotterdam, The Netherlands

A recent study tested the timing accuracy of several software solutions to implement computer-based experiments (Bridges et al. 2020). We discuss some conceptual and implementation issues in an attempt to bring more general awareness to what implementing timing-accurate experiments crucially relies on: (1) familiarizing oneself with the basic principles of stimulus presentation as well as the specifics of the used experiment control software, and (2) understanding that timing accuracy estimates should never be understood as a hard promise to simply rely on without additional verification in a specific lab setting. We believe that the raised issues should be considered in a discussion on how software for computer-based experiments should be tested. 
In the article "The timing mega-study: comparing a range of experiment generators, both lab-based and online" (Bridges et al., 2020), the authors attempted to compare the timing-accuracy of their own stimulus and response time recording software PsychoPy (Peirce, 2007) to that of a variety of other free as well as commercial software solutions. Most of the existing software in this field has been developed by university labs and researchers, which publish their work under open source licenses to give the users not only the essential freedom to run the software, but also the freedom to modify and further distribute the source code. Above that, most of these projects use public code repositories for collaborative software development and explicitly call for an active contribution of users to further development by submitting modifications. Unfortunately, developers of experiment control software have not been very successful during the last decades in establishing a collaborative agenda and common development effort towards a shared set of tools like it has been the case for most other areas of open source software. Reasons for this uncoordinated endeavour are many-folded and one might even argue that multiple independent projects result in a productive competition and are thus desired. Nevertheless, the current situation does call, in our view, for a systematic overview and evaluation of the existing experiment control software. We therefore appreciate the intended comparison of Bridges and colleagues (2020) and think that this idea could even serve as a starting point for regular systematic assessments of the open source tools commonly used in the fields of psychology and neuroscience. Importantly however, we believe that such an effort should be collaborative in nature, rather than competitive, where the criteria and the evaluation procedures need to be standardized, reproducible and based on a common agreement among developers and users. This is unfortunately not the case in the recent article, and upon closer examination of the described test procedures as well as the publicly available additional material, we identified several issues on both, the conceptual as well as the implementation level, which not only artificially produce the observed results and some surprising differences, but also highlight some crucial underlying issues worth discussing.

Another motivation for this commentary is that among the tested software solutions was the Python library Expyriment (Krause \& Lindemann, 2014) that has been developed and currently maintained by us. Importantly, unlike most of the tested software solutions which constitute full desktop applications with graphical user interfaces, Expyriment is a lightweight and flexible library for the Python programming language. Its purpose is not to provide researchers with an "experiment generator" application, but with the building blocks to programme timing-critical experiments in Python or to develop such applications themselves (e.g. OpenSesame, Mathôt et al. 2012; TrajTracker, Dotan et al., 2019). The robustness against erroneous usage is an important quality criterion for an experiment generator application. Such a criterion is however difficult to apply in a programming library, which gives users maximal flexibility but also responsibility for their implementation and final product. That is, a programming library can per definition merely guarantee particular outcome on the level of a function call and clearly defined use cases and not 
at the level of applications that are developed using this library. That said, in the tests of Expyriment reported by Bridges and colleagues (2020), the library appears to have performed very poorly and the authors even explicitly advise the reader against using it. We hence see it as our obligation to our users and fellow researchers to clarify that these results are neither representative nor generalizable.

Since timing accuracy is always the product of a complex interaction between experiment control software, the operating system, device drivers, and, crucially, the used hardware set up, it is frivolous and maybe even misleading to classify any software as accurate or inaccurate without taking into account the exact hardware setup. For instance, we discuss the details of using the Expyriment library to implement timing-accurate stimulus presentation and response recordings in the official documentation. Although Krause and Lindemann (2014) demonstrated the millisecond-accurate timing capabilities of Expyriment for certain hardware setup empirically, it has been highlighted that these findings can not be generalized and need to be verified for each hardware setup individually. We have even seen differences in the timing between laptops officially identical in construction but of different years of manufacture, and it is known that differences at the operating system and device driver level affect timing as well (Plant \& Quinlan, 2013).

Because of this, most experiment control software provide functionality to specifically test the timing accuracy of visual stimulus presentation on the target computers in the lab. The online documentation of Expyriment contains, in addition to that, concrete examples on how to optimize a system in case of a non-optimal test result. Unfortunately, despite the authors' claims of using each tested software solution according to their respective documentation, the publicly available test scripts, results and raw data of Bridges et al. (2020) show no signs that the authors attempted to verify that the system is set up to allow for timing-accurate visual stimulus presentation in the first place. To be precise, the reported visual duration times for Expyriment on Ubuntu Linux and MacOS are around half the refresh rate of the corresponding test systems $(60 \mathrm{~Hz})$. This indicates that code execution after stimulus presentation was not blocked until the vertical retrace has occurred - an important requirement for a system's ability to accurately report when exactly a stimulus was being drawn on the screen (see also Krause \& Lindemann, 2014). Moreover, the publicly available event files written by Expyriment during the reported tests contain the information that, despite the obvious inability of the system to block on the vertical retrace, the authors continued to test with the default method of attempting to block on the vertical retrace, rather than switching to the alternative method as suggested in the official documentation, which usually solves this issue on systems that fail to block with the default method. Finally, the article does not mention any other steps taken to verify, before testing, that the system has been set up as advised by the documentation of each tested software. At least for the Expyriment test, this led to results that are unfortunately not 
representative, and would have differed significantly, had the authors consulted the documentation, as one expects from a regular user of any software. In addition to the apparently non-optimised test setup, the publicly available implementations of the tests themselves unfortunately reveal further oversights that actively affected timing accuracy, such as performing time-consuming procedures (e.g. stimulus preloading) at timing-critical moments, without compensating for their computation time (in both the stimulus and response tests), or initiating the presentation of a new visual stimulus after the full interstimulus interval (e.g. $300 \mathrm{~ms}$ ) has already passed, while the goal seemed to have been having the new stimulus already on the display at the end of said interval (in the response test). It goes without saying that we would have gladly provided support, as we do for all our users, had we been contacted by the authors. Sadly, we were not approached to assist, and the authors went on to conclude that timing accuracy in Expyriment is generally hindered by its usage of the Pygame library (Pygame, 2021), which, according to them, has not been optimized for low-latency and high-precision timing, and recommend to not use Expyriment where precise stimulus/response timing is needed. In contrast to these claims, however, visual stimulus presentation in Expyriment is by default based on OpenGL (OpenGL, 2021) and both stimulus presentation as well as response monitoring can demonstrably be millisecond-accurate (Krause \& Lindemann, 2014, but see also https://docs.expyriment.org/Timing.html for further details).

Besides the concerns originating from inspecting the test implementation in Expyriment, we also identified more general issues in the used methods in Bridges et al. (2020) that probably also affected the results of other tested software solutions. For instance, the implementations of the response test differed significantly in aspects that directly affected the timing accuracy measurement: in some implementations (e.g. the one for Open Sesame; Mathôt et al.2012), the auditory stimulus was presented first, followed by the visual stimulus, followed by the TTL pulse, while in others (e.g. the one for Expyriment), the visual stimulus was presented first, followed by the TTL pulse, followed by the auditory stimulus. One explanation for these differences might lie in the authors' apparent unfamiliarity with presenting visual stimuli for an exact duration within the constraints of display refresh cycles in some of the tested software solutions that use a procedural rather than an event-driven programming paradigm (as discussed in their Materials and Methods section). Furthermore, the measurements of the timing accuracy of auditory stimulus presentation use vastly different settings across implementations. For instance, the audio buffer is set to 128 samples when testing PsychoPy, 1024 samples when testing OpenSesame, and 2048 samples when testing Expyriment. Since the size of the sample buffer is directly related to audio latency and jitter, this renders the reported results misleading at best.

Taken together, we hope that the issues we raise here can bring more general awareness to what implementing timing-accurate experiments crucially relies on. It is indispensable that experimenters 
familiarize themselves with the basic principles of stimulus presentation as well as the specifics of the used software solution, to be in a position to make informed decisions about the technical implementation and to achieve the best possible timing accuracy. Timing accuracy in any computer-based experiment software solution that runs on different systems can never be a hard promise to simply rely on, but refers to a general capability that a researcher needs to actively verify on each test system in question. While many software solutions provide the tools to easily verify the timing accuracy of visual stimulus presentation, verifying auditory stimulus presentation and response device timing often requires additional measurement equipment, such as the Black Box Toolkit (Plant et al., 2004) used by Bridges and colleagues (2020). We hence strongly encourage researchers to always verify visual stimulus timing accuracy before testing, and, in case no additional measurement equipment is available, to rely on external audio playback devices (e.g. professional sample players) and response devices that are know to be millisecond-accurate (i.e. not computer keyboards and mice; see also Plant \& Turner, 2009; Plant \& Quinlan, 2013).

Last, but not least, the raised issues should also make us rethink how software for computer-based experiments should be objectively tested and who should test them. We would advocate for the following: First, the authors of a specific software solution need to demonstrate the software's general capabilities under realistic scenarios. Second, researchers need to be provided with standardized test procedures and protocols to test for domain-specific requirements and the interactions between software and hardware in their labs. Finally, if meta-tests across software and hardware are to be made, we think it is important that these tests are done under comparable and reproducible conditions, according to the respective documentation as well as in consultancy and agreement with all involved developers. Ideally, such a joint effort would be coordinated by an independent party, in order to prevent any potential conflicts of interests. 


\section{References}

Bridges, D., Pitiot, A., MacAskill, M.R., Peirce, J.W. (2020). The timing mega-study: comparing a range of experiment generators, both lab-based and online. PeerJ 8:e9414. https://doi.org/10.7717/peeri.9414

Dotan D., Pinheiro-Chagas, P., Al Roumi, F., \& Dehaene S. (2019). Track it to crack it: Dissecting processing stages using finger tracking. Trends In Cognitive Sciences, 23(12), 1058-1070. https://doi.org/10.1016/j.tics.2019.10.002

Krause, F., \& Lindemann, O. (2014). Expyriment: A Python library for cognitive and neuroscientific experiments. Behavior Research Methods, 46(2), 416-428. https://doi.org/10.3758/s13428-013-0390-6

Mathôt, S., Schreij, D., \& Theeuwes, J. (2012). OpenSesame: An open-source, graphical experiment builder for the social sciences. Behavior Research Methods, 44(2), 314-324. doi:10.3758/s13428-011-0168-7

Peirce, J. W. (2007). PsychoPy: Psychophysics software in Python. Journal of Neuroscience Methods, 162, 8-13. https://doi.org/10.1016/j.jneumeth.2006.11.017

Plant, R.R., Hammond, N. \& Turner, G. (2004). Self-validating presentation and response timing in cognitive paradigms: How and why?. Behavior Research Methods, Instruments, \& Computers, 36, 291-303. https://doi.org/10.3758/BF03195575

Plant, R.R. \& Turner, G. (2009). Millisecond precision psychological research in a world of commodity computers: New hardware, new problems? Behavior Research Methods, 41, 598-614. https://doi.org/10.3758/BRM.41.3.598

Plant, R.R. \& Quinlan, P.T. (2013). Could millisecond timing errors in commonly used equipment be a cause of replication failure in some neuroscience studies? Cogn Affect Behav Neurosci, 13, 598-614. https://doi.org/10.3758/s13415-013-0166-6

Pygame. (2021). [Computer software]. Retrieved from https://www.pygame.org

PyOpenGL. (2021). [Computer software]. Retrieved from http://pyopengl.sourceforge.net 